\title{
Inner products of Bethe states as partial domain wall partition functions
}

\author{
Ivan Kostov ${ }^{a, 1}$ and Yutaka Matsuo ${ }^{b}$ \\ ${ }^{a}$ Institut de Physique Théorique, CNRS-URA 2306, \\ C.E.A.-Saclay, F-91191 Gif-sur-Yvette, France \\ ${ }^{b}$ Department of Physics, The University of Tokyo, \\ Hongo 7-3-1, Bunkyo-ku, Tokyo 113-0033, Japan \\ E-mail: ivan.kostov@cea.fr, matsuo@phys.s.u-tokyo.ac.jp
}

ABSTRACT: We study the inner product of Bethe states in the inhomogeneous periodic XXX spin- $1 / 2$ chain of length $L$, which is given by the Slavnov determinant formula. We show that the inner product of an on-shell $M$-magnon state with a generic $M$-magnon state is given by the same expression as the inner product of a $2 M$-magnon state with a vacuum descendent. The second inner product is proportional to the partition function of the six-vertex model on a rectangular $L \times 2 M$ grid, with partial domain-wall boundary conditions.

Keywords: Lattice Integrable Models, Bethe Ansatz

ARXIV EPRINT: 1207.2562

\footnotetext{
${ }^{1}$ Associate member of the Institute for Nuclear Research and Nuclear Energy, Bulgarian Academy of Sciences, 72 Tsarigradsko Chaussée, 1784 Sofia, Bulgaria.
} 


\section{Contents}

1 Introduction 1

2 Inner product of Bethe states in the inhomogeneous XXX 1/2 chain 2

3 Alternative expression for the inner product 5

3.1 Operator factorization formulas 5

3.2 The functional $\mathscr{A}_{\mathbf{u}}[f]$

3.3 A symmetric expression for the inner product 6

4 The Slavnov determinant as a pDWPF 4

5 Concluding remarks $\quad 11$

A Fermionic representation of the functionals $\mathscr{A}_{\mathrm{u}}^{ \pm}[f] \quad 12$

\section{Introduction}

The computation of the inner product of two Bethe wave functions is a necessary step on the way of obtaining the correlation functions in integrable models. An expansion formula for the scalar product of two generic Bethe states in the generalized $s u(2)$ model is due to Korepin [1]. The sum formula becomes increasingly difficult to tackle when the number of pseudoparticles becomes large. Simplifications occur when one of the states is on shell, i.e., when its rapidities satisfy the Bethe equations. In this case the inner product can be cast in the form of a determinant. A determinant formula for the norm of an on shell Bethe state was originally conjectured by Gaudin [2] and later proved by Korepin [1]. A determinant formula for the scalar product of one on shell and one generic Bethe states was found by N. Slavnov [3]. ${ }^{1}$ The Slavnov formula was used to obtain some correlation functions in the XXX and XXZ spin chains [4], and more recently in the computation of some correlation functions in the $\mathcal{N}=4$ supersymmetric Yang-Mills theory [6, 7, 9, 10].

If the two Bethe states are composed from $M$ pseudoparticles with rapidities $\mathbf{u}=$ $\left\{u_{1}, \ldots, u_{M}\right\}$ and $\mathbf{v}=\left\{v_{1}, \ldots, v_{M}\right\}$, the Slavnov inner product is given, up to a simple factor, by a $M \times M$ determinant. In [9, 10] one of the authors derived a a closed expression of the inner product for the XXX spin chain in the classical limit $M \rightarrow \infty$, in which the Bethe roots condense into several macroscopic strings. ${ }^{2}$ An intriguing feature of this expression is that it has the same form as the classical limit of the scalar product of a Bethe

\footnotetext{
${ }^{1}$ Previously, a representation of the inner product as a determinant of double size was found by Kirillov and Smirnov [5].

${ }^{2}$ The classical limit was studied in [11]; in the condensed matter physics it is known also as Sutherland limit [12].
} 
state with a vacuum descendent, obtained previously in [7], if one chooses the rapidities $\mathbf{w}$ of the Bethe state as $\mathbf{w}=\mathbf{u} \cup \mathbf{v}$.

In this paper we prove that the above is true also for finite $M$. We show that the Slavnov inner product $\langle\mathbf{v} \mid \mathbf{u}\rangle$ of two $M$-magnon states in a periodic inhomogeneous XXX chain with spin $1 / 2$ is equal to the inner product of a vacuum descendent with the $2 M$ magnon state $|\mathbf{u} \cup \mathbf{v}\rangle$, which is in turn proportional to the partition function of the six-vertex model with partial domain wall boundary conditions, studied recently in [13].

\section{Inner product of Bethe states in the inhomogeneous XXX 1/2 chain}

The XXX spin chain is characterized by an $R$-matrix $R_{12}(u, v)$ acting in the tensor product $V_{1} \otimes V_{2}$ of two copies of the target space. Up to a scalar factor, the $R$-matrix of the XXX spin chain is [14]

$$
R_{12}(u, v)=u-v+i P_{12}
$$

where $P_{12}$ is the permutation operator acting in the tensor product $V_{1} \otimes V_{2}$ of two copies of the target space. The inhomogeneous XXX spin chain of length $L$ is characterized by background parameters (impurities) $\mathbf{z}=\left\{z_{1}, \ldots, z_{L}\right\}$ associated with the $L$ sites of the chain. For the spin $1 / 2$ chain, the monodromy matrix $\mathcal{T}_{a}(u) \in \operatorname{End}\left(V_{a}\right)$ represents the product of the $R$-matrices along the spin chain,

$$
\mathcal{T}_{a}(u) \equiv R_{a 1}\left(u, z_{1}\right) R_{a 2}\left(u, z_{2}\right) \ldots R_{a L}\left(u, z_{L}\right)=\left(\begin{array}{cc}
\mathcal{A}(u) & \mathcal{B}(u) \\
\mathcal{C}(u) & \mathcal{D}(u)
\end{array}\right)
$$

The homogeneous XXX spin chain corresponds to the limit $z_{m} \rightarrow i / 2 .^{3}$

The matrix elements $\mathcal{A}, \mathcal{B}, \mathcal{C}, \mathcal{D}$ are operators in the Hilbert space $V=V_{1} \otimes \cdots \times V_{L}$ of the spin chain. The commutation relations between the elements of the monodromy matrix are determined by the RTT relation

$$
R_{12}(u-v) \mathcal{T}_{1}(u) \mathcal{T}_{2}(v)=\mathcal{T}_{2}(v) \mathcal{T}_{1}(u) R_{12}(u-v)
$$

which follows from the Yang-Baxter equation for $R$. In components,

$$
\begin{aligned}
\mathcal{A}(v) \mathcal{B}(u) & =\frac{u-v+i}{u-v} \mathcal{B}(u) \mathcal{A}(v)-\frac{i}{u-v} \mathcal{B}(v) \mathcal{A}(u), \\
\mathcal{D}(v) \mathcal{B}(u) & =\frac{u-v-i}{u-v} \mathcal{B}(u) \mathcal{D}(v)+\frac{i}{u-v} \mathcal{B}(v) \mathcal{D}(u), \\
{[\mathcal{C}(u), \mathcal{B}(v)] } & =\frac{i}{u-v}(\mathcal{A}(v) \mathcal{D}(u)-\mathcal{A}(u) \mathcal{D}(v)), \quad \text { etc. }
\end{aligned}
$$

As a consequence of $(2.3)$, the families of operators $\mathcal{B}(u), \mathcal{C}(u)$, as well as the transfer matrices

$$
\mathcal{T}(u) \equiv \operatorname{Tr}_{a}\left[\mathcal{T}_{a}(u)\right]=\mathcal{A}(u)+\mathcal{D}(u)
$$

are commuting.

\footnotetext{
${ }^{3}$ Sometimes $\theta_{j}=z_{j}-i / 2$ are referred to as inhomogeneity parameters. With this definitioon the homogeneous limit corresponds to $\theta_{j}=0$.
} 
In the Algebraic Bethe Ansatz, the Hilbert space has the structure of a Fock space generated by the action of the creation operators $\mathcal{B}(u)$ on the pseudo-vacuum $|\uparrow L\rangle=\mid \uparrow \uparrow$ $\therefore \uparrow \uparrow$, where all spins are oriented up. The pseudo-vacuum is an eigenstate for the diagonal elements $\mathcal{A}$ and $\mathcal{D}$ and is annihilated by $\mathcal{C}$ :

$$
(\mathcal{A}(u)-A(u))\left|\uparrow^{L}\right\rangle=(\mathcal{D}(u)-D(u))\left|\uparrow^{L}\right\rangle=\mathcal{C}(u)\left|\uparrow^{L}\right\rangle=0,
$$

where

$$
A(u)=\prod_{m=1}^{L}\left(u-z_{m}+i\right), \quad D(u)=\prod_{m=1}^{L}\left(u-z_{m}\right) .
$$

A creation/annihilation operator with $u \rightarrow \infty$ is the global $s u(2)$ lowering/raising operator,

$$
\frac{\mathcal{B}(u)}{D(u)} \simeq \frac{i}{u} \mathbb{S}^{-}, \quad \frac{\mathcal{C}(u)}{A(u)} \simeq \frac{i}{u} \mathbb{S}^{+} .
$$

The dual Bethe states are generated by the action of the $\mathcal{C}$-operators on the dual pseudo-vacuum $\left\langle\uparrow^{L}\right|=\langle\uparrow \uparrow \ldots \uparrow|$, which is annihilated by the $\mathcal{B}$-operators, and the hermitian conjugation can be defined as

$$
\mathcal{C}(u)=-\mathcal{B}^{\dagger}(\bar{u}) .
$$

The space of states is a closure of the linear span of all vectors of the form

$$
|\mathbf{u}\rangle=\prod_{j=1}^{M} \mathcal{B}\left(u_{j}\right)\left|\uparrow^{L}\right\rangle, \quad \mathbf{u}=\left\{u_{1}, \ldots u_{M}\right\}, \quad M \equiv \# \mathbf{u} .
$$

The operator $\mathcal{B}(u)$ can be viewed as a creation operator of a pseudoparticle (magnon) with momentum $p=\log \frac{u+i / 2}{u-i / 2}$. Such states are called generic, or off shell, Bethe states. The scalar product of two generic Bethe states, ${ }^{4}$

$$
\langle\mathbf{u} \mid \mathbf{v}\rangle=\left\langle\uparrow L\left|\prod_{k=1}^{M} \mathcal{C}\left(v_{k}\right) \prod_{j=1}^{M} \mathcal{B}\left(u_{k}\right)\right| \uparrow L\right\rangle,
$$

can be computed by applying the relations of the RTT algebra (2.3). For example, the scalar product of two one-magnon states is

$$
\langle v \mid u\rangle=\frac{i}{u-v}(A(v) D(u)-A(u) D(v)) .
$$

A Bethe state is an eigenvector of the transfer matrices [eq. (2.5)] if the rapidities $\mathbf{u}=\left\{u_{1}, \ldots u_{M}\right\}$ satisfies the on-shell condition, which is given by the Bethe equations [14]

$$
\prod_{k=1}^{M} \frac{u_{j}-u_{k}+i}{u_{j}-u_{k}-i}=-\prod_{m=1}^{L} \frac{u_{j}-z_{m}+i}{u_{j}-z_{m}}, \quad j=1, \ldots, M .
$$

\footnotetext{
${ }^{4}$ Here we abuse slightly the established notations, since with the convention $\mathcal{B}(u)^{\dagger}=-\mathcal{C}(\bar{u})$, the state dual to $|\mathbf{v}\rangle$ is $(-1)^{N}\langle\overline{\mathbf{v}}|$. In our notations the norm of a Bethe state is $\|\mathbf{u}\|^{2}=(-1)^{N}\langle\overline{\mathbf{u}} \mid \mathbf{u}\rangle$. If one of the states is on shell, then the set of its rapidities is invariant upon complex conjugation, and the inner products $(-1)^{N}\langle\overline{\mathbf{v}} \mid \mathbf{u}\rangle$ and $\langle\mathbf{v} \mid \mathbf{u}\rangle$ differ only by a phase factor.
} 
To avoid lengthy formulas, throughout this paper we will use systematically the following notations. For any set $\mathbf{w}=\left\{w_{j}\right\}_{j=1}^{N}$ of points in the complex plane, we define the Baxter polynomial

$$
Q_{\mathbf{w}}(u) \stackrel{\text { def }}{=} \prod_{j=1}^{N}\left(u-w_{j}\right), \quad N=\# \mathbf{w}
$$

as well as the rational function

$$
E_{\mathbf{u}}^{ \pm}(u) \stackrel{\text { def }}{=} \frac{Q_{\mathbf{u}}(u \pm i)}{Q_{\mathbf{u}}(u)}
$$

In these notations, the eigenvalue $T_{\mathbf{u}}(u)$ of the transfer matrix on the on-shell state $|\mathbf{u}\rangle$ is

$$
T_{\mathbf{u}}(u)=Q_{\mathbf{z}}(u+i) E_{\mathbf{u}}^{-}(u)+Q_{\mathbf{z}}(u) E_{\mathbf{u}}^{+}(u) .
$$

Another way to write the Bethe equations is as

$$
e^{2 i p_{\mathbf{u}}(u)}=-1, \quad u \in \mathbf{u}
$$

where the pseudomomentum $p_{\mathbf{u}}$, known also as counting function, is defined modulo $\pi$ by

$$
e^{2 i p_{\mathbf{u}}}=\frac{1}{E_{\mathbf{z}}^{+}} \frac{E_{\mathbf{u}}^{+}}{E_{\mathbf{u}}^{-}}
$$

As shown by Slavnov [3], when the state $|\mathbf{u}\rangle$ is on shell, the inner product with a generic Bethe state $\langle\mathbf{v}|$ is a determinant. One can write the Slavnov determinant formula as

$$
\begin{aligned}
\langle\mathbf{v} \mid \mathbf{u}\rangle & =\prod_{j=1}^{M} A\left(v_{j}\right) D\left(u_{j}\right) \mathscr{S}_{\mathbf{u}, \mathbf{v}}, \\
\mathscr{S}_{\mathbf{u}, \mathbf{v}} & =\frac{\operatorname{det}_{j k} \Omega\left(u_{j}, v_{k}\right)}{\operatorname{det}_{j k} \frac{1}{u_{j}-v_{k}+i}}
\end{aligned}
$$

where the Slavnov kernel $\Omega(u, v)$ is defined by

$$
\Omega(u, v)=t(u-v)-e^{2 i p_{\mathbf{u}}(v)} t(v-u), \quad t(u)=\frac{1}{u}-\frac{1}{u+i} .
$$

For example, eq. (2.20) gives for the inner product of two one-magnon states $(M=1)$

$$
\langle v \mid u\rangle=A(v) D(u) \mathscr{S}_{u, v}, \quad \mathscr{S}_{u, v}=i \frac{1-\frac{D(v)}{A(v)}}{u-v} .
$$

This expression indeed matches with the restriction of the general expression (2.12) when the rapidity $u$ is taken on shell, $D(u) / A(u)=1$. 


\section{Alternative expression for the inner product}

\subsection{Operator factorization formulas}

The Slavnov determinant (2.20) can be given a very convenient operator expression [9, 10], whose derivation we review below. We represent the Slavnov kernel $\Omega(u, v)$ as the result of the action of two difference operators on the Cauchy kernel $\frac{1}{u-v+i}$,

$$
\Omega(u, v)=\left(1-e^{2 i p_{\mathbf{u}}(v)} e^{i \partial / \partial v}\right)\left(e^{-i \partial / \partial u}-1\right) \frac{1}{u-v+i},
$$

and write the Slavnov determinant as the result of the action of $N$ pairs of difference operators to the Cauchy determinant ,

$$
\mathscr{S}_{\mathbf{u}, \mathbf{v}}=\frac{\prod_{v \in \mathbf{v}}\left(1-e^{2 i p_{\mathbf{u}}(v)} e^{i \partial / \partial v}\right) \prod_{u \in \mathbf{u}}\left(e^{-i \partial / \partial u}-1\right) \operatorname{det}_{j k} \frac{1}{u_{j}-v_{k}+i}}{\operatorname{det}_{j k} \frac{1}{u_{j}-v_{k}+i}} .
$$

Here, and in the following, the formulae contain products of difference operators and the ordering of the difference operators should be respected. The factors within each of the two blocks in the above formula commute, but the factors belonging to different blocks do not.

Now we apply the Cauchy identity

$$
\operatorname{det}_{j k} \frac{1}{u_{j}-v_{k}+i}=\frac{\prod_{j<k}\left(u_{j}-u_{k}\right) \prod_{j<k}\left(v_{k}-v_{j}\right)}{\prod_{j, k=1}^{M}\left(u_{j}-v_{k}+i\right)} \equiv \frac{\Delta_{\mathbf{u}} \Delta_{-\mathbf{v}}}{\Pi_{\mathbf{u}, \mathbf{v}}} .
$$

After repeated application of the obvious identities

$$
\begin{aligned}
e^{-i \partial / \partial u} \frac{1}{\Pi_{\mathbf{u}, \mathbf{v}}} & =E_{\mathbf{v}}^{+}(u) \frac{1}{\Pi_{\mathbf{u}, \mathbf{v}}} e^{-i \partial / \partial u} & & (u \in \mathbf{u}) \\
e^{i \partial / \partial v} \frac{1}{\Pi_{\mathbf{u}, \mathbf{v}}} & =E_{\mathbf{u}}^{-}\left(v_{j}\right) \frac{1}{\Pi_{\mathbf{u}, \mathbf{v}}} e^{i \partial / \partial v_{j}} & & (v \in \mathbf{v})
\end{aligned}
$$

and taking into account the expression (2.18) for the pseudomomentum, we write eq. (3.2) in a factorized operator form,

$$
\mathscr{S}_{\mathbf{u}, \mathbf{v}}=(-1)^{\# \mathbf{u}} \frac{1}{\Delta_{\mathbf{v}}} \prod_{v \in \mathbf{v}}\left(1-\frac{E_{\mathbf{u}}^{+}(v)}{E_{\mathbf{z}}^{+}(v)} e^{i \partial / \partial v}\right) \Delta_{\mathbf{v}} \cdot \frac{1}{\Delta_{\mathbf{u}}} \prod_{u \in \mathbf{u}}\left(1-E_{\mathbf{v}}^{+}(u) e^{-i \partial / \partial u}\right) \Delta_{\mathbf{u}}
$$

Here, we have to be careful in that the operator $\exp (i \partial / \partial v)$ acts on all factors on the right of it.

\subsection{The functional $\mathscr{A}_{\mathrm{u}}[f]$}

The two blocks of factors in the above operator expression have a similar form and suggest introducing the following quantity. For any set of points $\mathbf{u}=\left\{u_{j}\right\}_{j=1}^{M}$ in the complex plane and for any complex function $f(z)$, we define the functional

$$
\mathscr{A}_{\mathbf{u}}^{ \pm}[f] \stackrel{\text { def }}{=} \frac{1}{\Delta_{\mathbf{u}}} \prod_{u \in \mathbf{u}}\left(1-f(u) e^{ \pm i \partial / \partial u}\right) \Delta_{\mathbf{u}}
$$


Substituting $\Delta_{\mathbf{u}}=\operatorname{det}_{j k}\left(u_{j}^{k-1}\right)$, one can write this functional as a ratio of determinants [9, 10]

$$
\mathscr{A}_{\mathbf{u}}^{ \pm}[f]=\frac{\operatorname{det}_{j k}\left(u_{j}^{k-1}-f\left(u_{j}\right)\left(u_{j} \pm i\right)^{k-1}\right)}{\operatorname{det}_{j k}\left(u_{j}^{k-1}\right)},
$$

but for our purposes the operator representation (3.6) is more convenient.

The functional $\mathscr{A}_{\mathbf{u}}^{ \pm}[f]$ can be expanded as a sum of monomials associated with the partitions of the set $\mathbf{u}$ into two disjoint subsets,

$$
\mathscr{A}_{\mathbf{u}}^{ \pm}[f]=\sum_{\mathbf{u}^{\prime} \cup \mathbf{u}^{\prime \prime}=\mathbf{u}} \mathscr{A}_{\mathbf{u}^{\prime}, \mathbf{u}^{\prime \prime}}^{ \pm} \prod_{u^{\prime \prime} \in \mathbf{u}^{\prime \prime}}\left[-f\left(u^{\prime \prime}\right)\right], \quad \mathscr{A}_{\mathbf{u}^{\prime}, \mathbf{u}^{\prime \prime}}^{ \pm}=\prod_{u^{\prime \prime} \in \mathbf{u}^{\prime \prime}} E_{\mathbf{u}^{\prime}}^{ \pm}\left(u^{\prime \prime}\right)
$$

Under this form, the functional $\mathscr{A}_{\mathbf{u}}^{ \pm}[f]$ appeared previously in ref. [7]. If the function $f(u)$ depends implicitly on $\mathbf{u}$, we define the functional $\mathscr{A}_{\mathbf{u}}^{ \pm}[f]$ so that it is given by the same expansion (3.8).

The operators $\mathscr{A}_{\mathbf{u}}^{+}[f]$ and $\mathscr{A}_{\mathbf{u}}^{-}[f]$ are related by the functional identities

$$
\begin{array}{lll}
\mathscr{A}_{\mathbf{u}}^{-}[f]=\mathscr{A}_{\mathbf{u}}^{+}[1 / f] \prod_{u \in \mathbf{u}}[-f(u)], & \mathscr{A}_{\mathbf{u}}^{+}[f]=\mathscr{A}_{\mathbf{u}}^{-}[1 / f] \prod_{u \in \mathbf{u}}[-f(u)], \\
\mathscr{A}_{\mathbf{u}}^{-}[f]=\mathscr{A}_{\mathbf{u}}^{+}\left[-\frac{E_{\mathbf{u}}^{-}}{E_{\mathbf{u}}^{+}} f\right], & \mathscr{A}_{\mathbf{u}}^{+}[f]=\mathscr{A}_{\mathbf{u}}^{-}\left[-\frac{E_{\mathbf{u}}^{+}}{E_{\mathbf{u}}^{-}} f\right] .
\end{array}
$$

The first pair of identities was proved in $[9,10]$. Here we give the proof of the second pair. Proof of eq. (3.10): We transform the coefficients $\mathscr{A}_{\mathbf{u}^{\prime}, \mathbf{u}^{\prime \prime}}^{-}$of the expansion (3.8) of $\mathscr{A}_{\mathbf{u}}^{-}[f]$ as

$$
\mathscr{A}_{\mathbf{u}^{\prime}, \mathbf{u}^{\prime \prime}}^{-}=\prod_{u^{\prime \prime} \in \mathbf{u}^{\prime \prime}} E_{\mathbf{u}^{\prime}}^{-}\left(u^{\prime \prime}\right)=\prod_{u^{\prime \prime} \in \mathbf{u}^{\prime \prime}} \frac{E_{\mathbf{u}^{\prime}}^{-}\left(u^{\prime \prime}\right)}{E_{\mathbf{u}^{\prime}}^{+}\left(u^{\prime \prime}\right)} E_{\mathbf{u}^{\prime}}^{+}\left(u^{\prime \prime}\right)=(-1)^{\# \mathbf{u}^{\prime \prime}} \prod_{u^{\prime \prime} \in \mathbf{u}^{\prime \prime}} \frac{E_{\mathbf{u}}^{-}\left(u^{\prime \prime}\right)}{E_{\mathbf{u}}^{+}\left(u^{\prime \prime}\right)} E_{\mathbf{u}^{\prime}}^{+}\left(u^{\prime \prime}\right)
$$

where we used the property $E_{\mathbf{u}}^{ \pm}=E_{\mathbf{u}^{\prime}}^{ \pm} E_{\mathbf{u}^{\prime \prime}}^{ \pm}$, as well as the obvious identity

$$
\prod_{u^{\prime \prime} \in \mathbf{u}^{\prime \prime}} \frac{E_{\mathbf{u}^{\prime \prime}}^{-}\left(u^{\prime \prime}\right)}{E_{\mathbf{u}^{\prime \prime}}^{+}\left(u^{\prime \prime}\right)}=(-1)^{\# \mathbf{u}^{\prime \prime}}
$$

Summing over all partitions, we obtain the expansion of the functional $\mathscr{A}^{+}[g]$, with $g=$ $-E_{\mathbf{u}}^{-} / E_{\mathbf{u}}^{+} f$.

\subsection{A symmetric expression for the inner product}

We can associate with the functional $\mathscr{A}_{\mathbf{u}}^{ \pm}[f]$ a difference operator $\hat{\mathscr{A}}_{\mathbf{u}}^{ \pm}[f]$ acting on the functions on the set $\mathbf{u}$, by replacing $f(u) \rightarrow f(u) e^{ \pm i \partial / \partial u}$ for all $u \in \mathbf{u}$. The operator $\hat{\mathscr{A}}_{\mathbf{u}}^{ \pm}[f]$ is well defined if the function $f(u)$ does not depend implicitly on the variables $\mathbf{u}$. The c-functional $\mathscr{A}_{\mathbf{u}}[f]$ is the result of the action of the operator functional $\hat{\mathscr{A}}_{\mathbf{u}}^{ \pm}[f]$ on the constant function 1 ,

$$
\mathscr{A}_{\mathbf{u}}^{ \pm}[f]=\hat{\mathscr{A}}_{\mathbf{u}}^{ \pm}[f] \cdot 1 .
$$

Then the expression [eq. (3.5)] for the inner product can be laid down in terms of the functionals $\mathscr{A}_{\mathbf{u}}^{-}$and $\hat{\mathscr{A}}_{\mathbf{v}}^{+}$as

$$
\mathscr{S}_{\mathbf{u}, \mathbf{v}}=(-1)^{\# \mathbf{u}} \hat{\mathscr{A}}_{\mathbf{v}}^{+}\left[E_{\mathbf{u}}^{+} / E_{\mathbf{z}}^{+}\right] \cdot \mathscr{A}_{\mathbf{u}}^{-}\left[E_{\mathbf{v}}^{+}\right] .
$$

eq. (3.13) is equivalent to the operator factorization formula derived in $[9,10]$. 
Below we give an alternative expression for the inner product, symmetric with respect to the sets of rapidities $\mathbf{u}$ and $\mathbf{v}$. Define the functional

$$
\tilde{\mathscr{S}}_{\mathbf{u}, \mathbf{v}} \stackrel{\text { def }}{=}(-1)^{\# \mathbf{u}} \hat{\mathscr{A}}_{\mathbf{v}}^{+}\left[E_{\mathbf{u}}^{+} / E_{\mathbf{z}}^{+}\right] \cdot \mathscr{A}_{\mathbf{u}}^{+}\left[E_{\mathbf{v}}^{+} / E_{\mathbf{z}}^{+}\right]=\tilde{\mathscr{S}}_{\mathbf{v}, \mathbf{u}} \cdot
$$

If the rapidities $\mathbf{u}$ in eq. (3.13) are assumed to be on shell, we can apply the first identity (3.10) to transform the second factor on the r.h.s. as

$$
\mathscr{A}_{\mathbf{u}}^{-}\left[E_{\mathbf{v}}^{+}\right] \stackrel{(3.10)}{=} \mathscr{A}_{\mathbf{u}}^{+}\left[-\frac{E_{\mathbf{u}}^{-}}{E_{\mathbf{u}}^{+}} E_{\mathbf{v}}^{+}\right] \stackrel{(2.18)}{=} \mathscr{A}_{\mathbf{u}}^{+}\left[-e^{2 i p_{\mathbf{u}}} \frac{E_{\mathbf{v}}^{+}}{E_{\mathbf{z}}^{+}}\right] \stackrel{(2.17)}{=} \mathscr{A}_{\mathbf{u}}^{+}\left[\frac{E_{\mathbf{v}}^{+}}{E_{\mathbf{z}}^{+}}\right]
$$

Therefore we can identify

$$
\mathscr{S}_{\mathbf{u}, \mathbf{v}}=\tilde{\mathscr{S}}_{\mathbf{u}, \mathbf{v}} \text { for } \mathbf{u} \text { on shell. }
$$

The functional $\tilde{\mathscr{S}}_{\mathbf{u}, \mathbf{v}}$ can be simplified further. It is given, up to a sign, by the functional $\mathscr{A}_{\mathbf{w}}\left[1 / E_{\mathbf{z}}^{+}\right]$, with $\mathbf{w}=\mathbf{u} \cup \mathbf{v}$ :

$$
\tilde{\mathscr{S}}_{\mathbf{u}, \mathbf{v}}=(-1)^{\# \mathbf{u}} \mathscr{A}_{\mathbf{u} \cup \mathbf{v}}^{+}\left[1 / E_{\mathbf{z}}^{+}\right] .
$$

Proof: Using the definition (3.6) and decomposing

$$
\Delta_{\mathbf{u} \cup \mathbf{v}}=\Delta_{\mathbf{u}} \Delta_{\mathbf{v}} \prod_{u \in \mathbf{u}, v \in \mathbf{v}}(u-v),
$$

we write $\mathscr{A}_{\mathbf{u} \cup \mathbf{v}}^{+}\left[1 / E_{\mathbf{z}}^{+}\right]$as a product of two operators, one depending explicitly only on the set $\mathbf{u}$, and the other depending on the set $\mathbf{v}$ :

$$
\begin{aligned}
\mathscr{A}_{\mathbf{u} \cup \mathbf{v}}^{+}\left[1 / E_{\mathbf{z}}^{+}\right]= & \frac{1}{\Delta_{\mathbf{v}}} \prod_{v \in \mathbf{v}}\left(1-\frac{E_{\mathbf{u}}^{+}(v)}{E_{\mathbf{z}}^{+}(v)} e^{i \partial / \partial v}\right) \Delta_{\mathbf{v}} \cdot \frac{1}{\Delta_{\mathbf{u}}} \prod_{u \in \mathbf{u}}\left(1-\frac{E_{\mathbf{v}}^{+}(u)}{E_{\mathbf{z}}^{+}(u)} e^{i \partial / \partial u}\right) \Delta_{\mathbf{u}} \\
& =\hat{\mathscr{A}}_{\mathbf{v}}^{+}\left[E_{\mathbf{u}}^{+} / E_{\mathbf{z}}^{+}\right] \cdot \mathscr{A}_{\mathbf{u}}^{+}\left[E_{\mathbf{v}}^{+} / E_{\mathbf{z}}^{+}\right] \\
& =(-1)^{\# \mathbf{u}} \tilde{\mathscr{S}}_{\mathbf{u}, \mathbf{v}} .
\end{aligned}
$$

As an example, apply this formula for the inner product [eq. (2.12)] of two one-magnon states:

$$
\begin{aligned}
\tilde{\mathscr{S}}_{u, v}=\mathscr{A}_{\{u, v\}}^{+}\left[1 / E_{\mathbf{z}}^{+}\right] & =1-E_{v}^{+}(u) / E_{\mathbf{z}}^{+}(u)-E_{u}^{+}(v) / E_{\mathbf{z}}^{+}(v)+1 / E_{\mathbf{z}}^{+}(u) E_{\mathbf{z}}^{+}(v) \\
& =\left(1-1 / E_{\mathbf{z}}^{+}(u)\right)\left(1-1 / E_{\mathbf{z}}^{+}(v)\right)+i \frac{1 / E_{\mathbf{z}}^{+}(u)-1 / E_{\mathbf{z}}^{+}(v)}{u-v} .
\end{aligned}
$$

If the rapidity $u$ is on shell, then is $E_{\mathbf{z}}^{+}=1$ and the first term disappears, while the second gives the inner product.

A representation of the r.h.s. of (3.17) as a Fock expectation value of chiral fermions is given in the appendix. 


\section{The Slavnov determinant as a pDWPF}

The inner product of two $M$-magnon states can be thought of as as a partition function of the six-vertex model on a $2 M \times L$ rectangular grid, with particular boundary conditions. The $R$-matrix $R(u, v)$ [eq. (2.1)] can be graphically represented as two intersecting segments, a horizontal one carrying a rapidity $u$ and a vertical one carrying rapidity $v$,

$$
\mathcal{R}(u-v)=u \frac{\left.{ }^{j}\right|^{l} k}{m}=(u-v) \delta_{j k} \delta_{l m}+i \delta_{j l} \delta_{k m} .
$$

The two halves of each segment are labeled by $s u(2)$ indices, which are represented in a standard way by arrows. Then the six non-vanishing elements of the $R$-matrix correspond to the vertices of the six-vertex model in the rational limit [16], depicted in figure 1, with Boltzmann weights

$$
a(u, v)=u-v+i, \quad b(u, v)=u-v, \quad c(u, v)=i .
$$

The operators $\mathcal{B}(u)$ and $\mathcal{C}(u)$ are graphically represented in figure 2 . The inner product [eq. (2.11)] can be identified as the partition function of the six-vertex model defined on a $2 M \times L$ rectangular grid, shown in figure 3 . The partition function is a sum over all possible ways to associate arrows with the internal links, so that at each site of the lattice the number of the incoming arrows equals the number of the outgoing arrows. The boundary conditions on the arrow configurations are the following: on the two vertical boundaries, the lower half of the arrows point outwards, while the upper half point inwards. On the two horizontal boundaries all arrows point upwards. For example, the scalar product of two one-magnon states, eq. (2.12), is represented as

$$
\langle v \mid u\rangle=\sum_{k=1}^{L}
$$

The identity (3.16) means that the Slavnov inner product has a second interpretation as a partition function of the six-vertex model. Before formulating the correspondence, we give a brief recollection of the (partial) domain wall partition functions.

The domain wall partition function, DWPF, is the partition function of the six-vertex model on a square grid with domain wall boundary conditions, DWBC $[1,17]$. The DWBC restrict the arrows on the edges forming the two horizontal boundaries to point outwards, while the arrow on the edges forming the two vertical boundaries point inwards, as shown in figure 4 . The weights depend on the rapidity variables $\mathbf{w}=\left\{w_{1}, \ldots, w_{N}\right\}$ and $\mathbf{z}=$ $\left\{z_{1}, \ldots, z_{N}\right\}$, associated respectively with the horizontal and with the vertical lines. As shown by Izergin $[18,19]$, the DWPF can be expressed, up to a factor which can be 


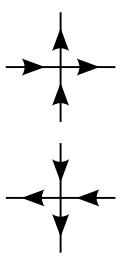

$a$

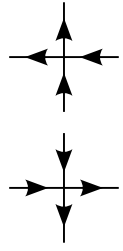

b

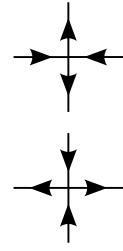

$c$
Figure 1. Graphical representation of the non-vanishing elements of the $R$ matrix represented by the vertices of the six-vertex model.

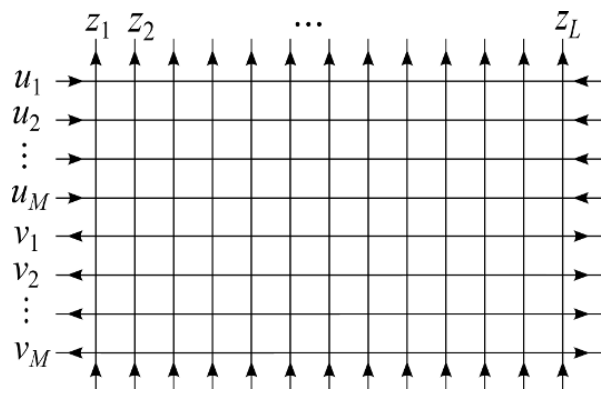

Figure 3. Graphical representation of the inner product $\langle\mathbf{v} \mid \mathbf{u}\rangle$ as a six-vertex partition function on a rectangular grid.

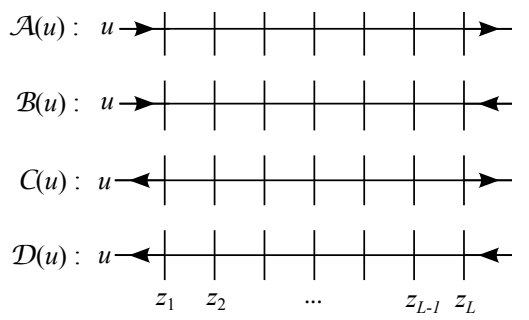

Figure 2. Graphical representation of the elements of the monodromy matrix

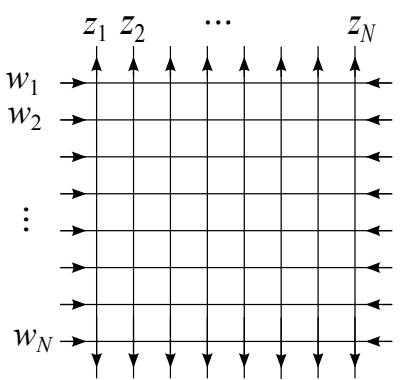

Figure 4. The domain wall boundary conditions (DWBC).

eliminated by a renormalization of the six-vertex weights, as a determinant ${ }^{5}$

$$
\mathscr{Z}_{\mathbf{w}, \mathbf{z}}=\frac{\operatorname{det}_{j k} t\left(w_{j}-z_{k}\right)}{\operatorname{det}_{j k} \frac{1}{w_{j}-z_{k}+i}}, \quad t(u)=\frac{1}{u}-\frac{1}{u+i} .
$$

The Gaudin-Izergin determinant (4.4) is a particular case $M=L=N, \mathbf{u}=\mathbf{w}, \mathbf{v}=\mathbf{z}$, of the Slavnov determinant (2.20). Indeed, if $\mathbf{v}=\mathbf{z}$, the second term in the Slavnov matrix $\Omega$ vanishes, $\Omega(u, v)=t(u-v)$. Then the first factor in (3.5) is equal to 1 , and we find [10]

$$
\mathscr{Z}_{\mathbf{w}, \mathbf{z}}=(-1)^{N} \mathscr{A}_{\mathbf{w}}^{-}\left[E_{\mathbf{z}}^{+}\right]=(-1)^{N} \mathscr{A}_{\mathbf{z}}^{+}\left[E_{\mathbf{w}}^{-}\right], \quad \# \mathbf{w}=\# \mathbf{z}=N .
$$

eq. (4.5) can be derived also directly from the definition (4.4), proceeding in the same way as we did in the case of the Slavnov determinant. In this derivation one does not need to assume that the rapidities $\mathbf{w}$ are on shell.

The quantities named in [13] partial domain wall partition functions, or pDWPF, are defined on a rectangular grid $n \times N$, where $1 \leq n \leq N$. The boundary conditions are the same as the DWBC except for the bottom segment of the boundary. On the $N \times N$ square grid, the positions of the bottom arrows are fixed, once we have imposed the DWBC on

\footnotetext{
${ }^{5}$ For the first time the ratio of determinants (4.4) appeared in the works of M. Gaudin [2, 20] as the scalar product of two Bethe wave functions for a Bose gas with point-like interaction on an infinite line.
} 


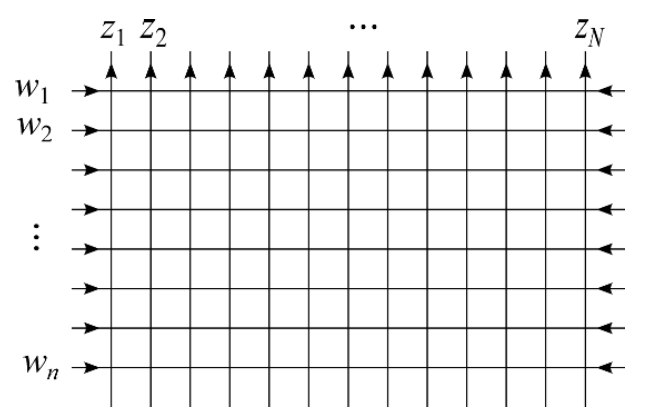

Figure 5. The partial domain wall boundary conditions (pDWBC). The boundary arrows on the top point outwards, those on the vertical boundaries point inwards, and those on the bottom segment are free.

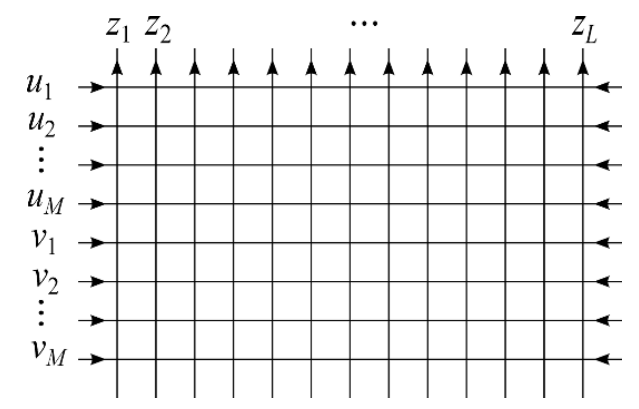

Figure 6. The pDWBC relevant for the Slavnov inner product.

the left, the right and the top segments of the boundary. When $n<N$, this is no more the case. The partial DWBC, depicted in figure 5, do not impose any restriction to the positions of the arrows on the bottom segment, and the bottom arrow configurations are summed over, just as those for the bulk segments. We will denote the pDWPF again by $\mathscr{Z}_{\mathbf{w}, \mathbf{z}}$, with $1 \leq \# \mathbf{w} \leq \# \mathbf{z}$. When this is needed by the context, we will denote by $\mathbf{w}_{n}$ a set of rapidities $\mathbf{w}$ with cardinality $\# \mathbf{w}=n$.

The pDWPF can be obtained from the DWPF by sending the rapidities $w_{n+1}, \ldots, w_{N}$, associated with the bottom $N-n$ rows, sequentially to infinity. Let $\mathbf{u}_{n}$ be the subset of the first $n$ rapidities in $\mathbf{u}_{N}=\left\{u_{1}, \ldots, u_{N}\right\}$. Then the result of sending the remaining $N-n$ rapidities to infinity is $[10,13]$

$$
\mathscr{Z}_{\mathbf{u}_{n}, \mathbf{z}_{N}}=\lim _{u_{1} \rightarrow \infty} \frac{u_{1}}{i} \ldots \lim _{u_{N} \rightarrow \infty} \frac{u_{N}}{i} \mathscr{Z}_{\mathbf{u}_{N}, \mathbf{z}_{N}}=(-1)^{n}(N-n) ! \mathscr{A}_{\mathbf{u}_{n}}^{-}\left[E_{\mathbf{z}_{N}}^{+}\right] .
$$

Applying the second identity (3.9) to the r.h.s. of (3.17), we can relate the functional $\tilde{\mathscr{S}}_{\mathbf{u}, \mathbf{v}}$ to the partial domain wall partition function $\mathcal{Z}_{\mathbf{u} \cup \mathbf{v}, \mathbf{z}}$ defined on the rectangular $2 N \times L$ grid, as the one shown in figure 6 , where $N=\# \mathbf{u}$ and $L=\# \mathbf{z}$,

$$
\tilde{\mathscr{S}}_{\mathbf{u}, \mathbf{v}}=(-1)^{\# \mathbf{u}} \frac{\mathscr{Z}_{\mathbf{u} \cup \mathbf{v}, \mathbf{z}}}{\mathcal{N}_{\mathbf{u} \cup \mathbf{v}, \mathbf{z}}} \quad, \quad \mathcal{N}_{\mathbf{w}, \mathbf{z}} \stackrel{\text { def }}{=}(\# \mathbf{z}-\# \mathbf{w}) ! \prod_{w \in \mathbf{w}} E_{\mathbf{z}}^{+}(w) .
$$

Another way to write the proportionality factor is as

$$
\mathcal{N}_{\mathbf{w}, \mathbf{z}}=(\# \mathbf{z}-\# \mathbf{w}) ! \prod_{w \in \mathbf{w}} \frac{A(w)}{D(w)}
$$

The proof eq. (4.7) is surprisingly simple. First we transform the representation (4.6), using the first of the functional identities (3.9),

$$
\mathscr{Z}_{\mathbf{u} \cup \mathbf{v}, \mathbf{z}}=(L-2 M) ! \mathscr{A}_{\mathbf{u} \cup \mathbf{v}}^{-}\left[E_{\mathbf{z}}^{+}\right]=\mathcal{N}_{\mathbf{u} \cup \mathbf{v}, \mathbf{z}} \mathscr{A}_{\mathbf{u} \cup \mathbf{v}}^{+}\left[1 / E_{\mathbf{z}}^{+}\right] .
$$

This concludes the proof of (4.7). 


\section{Concluding remarks}

In this paper, we derived an expression for the inner product of an $M$-magnon Bethe eigenstate and an $M$-magnon generic state in the inhomogeneous periodic XXX chain of length $\# \mathbf{z}=L$, which is completely symmetric in the union of the two sets of rapidity parameters:

$$
\tilde{\mathscr{S}}_{\mathbf{u}, \mathbf{v}}=(-1)^{\# \mathbf{u}} \mathscr{A}_{\mathbf{u} \cup \mathbf{v}}^{+}\left[1 / E_{\mathbf{z}}^{+}\right]
$$

The functional $\tilde{\mathscr{S}}_{\mathbf{u}, \mathbf{v}}$ represents a determinant $2 M \times 2 M$ and is given essentially by the partition function with domain boundary conditions on a $L \times L$ square grid, with $L-2 M$ of rapidities sent to infinity. The functional $\tilde{\mathscr{S}}_{\mathbf{u}, \mathbf{v}}$ coincides with the original Slavnov product (3.13) if the rapidities $\mathbf{u}$ are on shell, and for periodic boundary conditions (no twist in the Bethe equations). In general, $\tilde{\mathscr{S}}_{\mathbf{u}, \mathbf{v}}$ and $\mathscr{S}_{\mathbf{u}, \mathbf{v}}$ are two distinct functionals.

An immediate application of the representation (5.1) in the study of the semiclassical limit of the three-point function of long trace operators in the $s u(2)$ sector $\mathcal{N}=4 \mathrm{SYM}$, formulated in refs. [6, 7]. Using the determinant representation given in [8], a closed expression for the structure constant for three non-protected operators was obtained in refs. $[9,10]$ as a generalization of the result for one-protected and two non-protected operators operators found in ref. [7]. The classical limit of the functionals $\mathscr{S}_{\mathbf{u}, \mathbf{v}}$ and $\tilde{\mathscr{S}}_{\mathbf{u}, \mathbf{v}}$ is the same, but if one is interested in the subleading terms, second functional is much more convenient to deal with. On the other hand, the expression (5.1) with generic inhomogeneity parameters can be used to reproduce the higher orders in the weak coupling expansion of the structure constant, as it has been argued in [30, 31].

The alternative representation of the inner product (2.11), found in this paper, has a natural interpretation on terms of the Fock space for the Algebraic Bethe Ansatz. The functional $\tilde{\mathscr{S}}_{\mathbf{u}, \mathbf{v}}$ is proportional to the inner product

$$
\begin{aligned}
\tilde{\mathscr{S}}_{\mathbf{u}, \mathbf{v}} & \sim\left\langle\downarrow^{L}\left|\left(\mathbb{S}^{-}\right)^{L-2 M} \prod_{j=1}^{M} \mathcal{B}\left(v_{j}\right) \prod_{j=1}^{M} \mathcal{B}\left(u_{j}\right)\right| \uparrow^{L}\right\rangle \\
& \sim\left\langle\uparrow L\left|\prod_{j=1}^{M} \mathcal{C}\left(v_{j}\right) \prod_{j=1}^{M} \mathcal{C}\left(u_{j}\right)\left(\mathbb{S}^{+}\right)^{L-2 M}\right| \downarrow^{L}\right\rangle .
\end{aligned}
$$

The second functional has the same structure as the result of a particle-hole transformation on the ket vector on the r.h.s. of the original inner product [eq. (2.11)]. In case of a nonzero twist, the dual rapidities are different than the original rapidities [29]. In the case we are considering, $M$ of the dual rapidities coincide with the original ones, while the the rest $L-2 M$ of them go to infinity. This can be justified by the following simple argument. ${ }^{6}$ For zero twist, the global $s u(2)$ symmetry is not broken and the Bethe states is a direct sum of states with given spin, which are eigenspaces of the transfer matrix. The states corresponding to the same solution of the Bethe equations, belong to the same $s u(2)$ multiplet [32]. Therefore the Bethe eigenstates $\prod_{u \in \mathbf{u}} \mathcal{B}(u)\left|\uparrow^{L}\right\rangle$ and $\prod_{u \in \mathbf{u}} \mathcal{C}(u)\left|\downarrow^{L}\right\rangle$ must

\footnotetext{
${ }^{6}$ We thank D. Serban for suggesting to us this argument.
} 
be related by the action of a global raising operator, which must be $\left(\mathbb{S}^{+}\right)^{L-2 M}$, since the first state has $S^{z}=\frac{1}{2} L-M$, while the second state has $S^{z}=M-\frac{1}{2} L$. Thus we have

$$
\prod_{j=1}^{M} \mathcal{C}\left(u_{j}\right)\left(\mathbb{S}^{+}\right)^{L-2 M}\left|\downarrow^{L}\right\rangle \sim \prod_{j=1}^{M} \mathcal{B}\left(u_{j}\right)\left|\uparrow^{L}\right\rangle,
$$

where the proportionality sign means that the two states are equal up a c-function of the rapidities. In this sense, our main result is the computation of the factor of proportionality.

Note added. After the preparation of the manuscript, we learned about the publication [33], which is along the same lines as our work.

\section{Acknowledgments}

I.K. thanks O. Foda, D. Serban, A. Sever and F. Smirnov for useful discussions. YM is grateful to the hospitality of the collegues in Saclay during his stay. This research has received funding from the [European Union] Seventh Framework Programme [FP7People-2010-IRSES] under grant agreement $N^{o}$ 269217, the PHC SAKURA 2012, Projet $\mathrm{N}^{o}$ 27588UASakura and corresponding Grant from Japan. YM is partially supported by Grant-in-Aid (KAKENHI \#20540253) from MEXT Japan.

\section{A Fermionic representation of the functionals $\mathscr{A}_{\mathbf{u}}^{ \pm}[f]$}

The functionals $\mathscr{A}_{\mathbf{u}}^{-}\left[E_{\mathbf{z}}^{+}\right]$and $\mathscr{A}_{\mathbf{u}}^{+}\left[1 / E_{\mathbf{z}}^{+}\right]$have natural fermionic representations. Introduce a chiral Neveu-Schwarz fermion living in the rapidity complex plane and having mode expansion

$$
\psi(u)=\sum_{r \in \mathbb{Z}+\frac{1}{2}} \psi_{r} u^{-r-\frac{1}{2}}, \quad \bar{\psi}(u)=\sum_{r \in \mathbb{Z}+\frac{1}{2}} \bar{\psi}_{r} u^{r-\frac{1}{2}} .
$$

The fermion modes are assumed to satisfy the anticommutation relations

$$
\left[\bar{\psi}_{r}, \bar{\psi}_{r^{\prime}}\right]_{+}=\left[\psi_{r}, \psi_{r^{\prime}}\right]_{+}=0, \quad\left[\psi_{r}, \bar{\psi}_{r^{\prime}}\right]_{+}=\delta_{r, r^{\prime}}
$$

and the left/right vacuum states are defined by

$$
\langle 0| \psi_{-r}=\langle 0| \bar{\psi}_{r}=0 \text { and } \quad \psi_{r}|0\rangle=\bar{\psi}_{-r}|0\rangle=0, \quad \text { for } r>0 .
$$

The operator $\bar{\psi}_{r}$ creates a particle (or annihilates a hole) with mode number $r$ and the operator $\psi_{r}$ annihilates a particle (or creates a hole) with mode number $r$. The particles carry charge 1 , while the holes carry charge -1 . The charge zero vacuum states (A.3) are obtained by filling the Dirac sea up to level zero. The left vacuum states with integer charge $\pm N$ are constructed as

$$
\langle N|= \begin{cases}\langle 0| \psi_{\frac{1}{2}} \ldots \psi_{N-\frac{1}{2}} & \text { if } N>0 \\ \langle 0| \bar{\psi}_{-\frac{1}{2}} \ldots \bar{\psi}_{-N+\frac{1}{2}} & \text { if } N<0\end{cases}
$$


Any correlation function of the operators (A.1) is a determinant of two-point correlators

$$
\langle 0|\psi(u) \bar{\psi}(v)| 0\rangle=\frac{1}{u-v} .
$$

The following formulae is easily established. Let $\# \mathbf{u}=n$ and $\# \mathbf{z}=N$. Then

$$
\begin{aligned}
\mathscr{A}_{\mathbf{u}}^{+}\left[1 / E_{\mathbf{z}}^{+}\right] & =\frac{\left\langle N-n\left|\prod_{j=1}^{n}\left[\psi\left(u_{j}\right)-\psi\left(u_{j}+i\right)\right] \prod_{k=1}^{N} \bar{\psi}\left(z_{k}\right)\right| 0\right\rangle}{\left\langle N-n\left|\prod_{j=1}^{n} \psi\left(u_{j}\right) \prod_{k=1}^{N} \bar{\psi}\left(z_{k}\right)\right| 0\right\rangle}, \\
\mathscr{A}_{\mathbf{u}}^{-}\left[E_{\mathbf{z}}^{+}\right] & =\frac{\left\langle N-n\left|\prod_{j=1}^{n}\left[\psi\left(u_{j}\right)-\psi\left(u_{j}+i\right)\right] \prod_{k=1}^{N} \bar{\psi}\left(z_{k}\right)\right| 0\right\rangle}{\left\langle N-n\left|\prod_{j=1}^{n} \psi\left(u_{j}+i\right) \prod_{k=1}^{N} \bar{\psi}\left(z_{k}\right)\right| 0\right\rangle} .
\end{aligned}
$$

Eq. (A.6) gives a convenient representation of the pDWPF and the Slavnov scalar product in terms of free chiral fermions. Other fermionic representations have been proposed in [21-23] for the Slavnov product and in [24-26] for the Gaudin-Izergin determinant, see also the review paper [27].

Open Access. This article is distributed under the terms of the Creative Commons Attribution License which permits any use, distribution and reproduction in any medium, provided the original author(s) and source are credited.

\section{References}

[1] V. Korepin, Calculation of norms of Bethe wave functions, Commun. Math. Phys. 86 (1982) 391 [INSPIRE].

[2] M. Gaudin, La fonction d'onde de Bethe, Masson, Paris, France (1983).

[3] N.A. Slavnov, Calculation of scalar products of wave functions and form factors in the framework of the algebraic Bethe ansatz, Theor. Math. Phys. 79 (1989) 502.

[4] N. Kitanine, J. Maillet, N. Slavnov and V. Terras, On the algebraic Bethe ansatz approach to the correlation functions of the XXZ spin-1/2 Heisenberg chain, hep-th/0505006 [INSPIRE].

[5] A.N. Kirillov and F.A. Smirnov, Formfactors in the SU(2) - invariant thirring model, Zap. Nauchn. Semin. LOMI, 164 (1987) 67.

[6] J. Escobedo, N. Gromov, A. Sever and P. Vieira, Tailoring Three-Point Functions and Integrability, JHEP 09 (2011) 028 [arXiv:1012.2475] [INSPIRE].

[7] N. Gromov, A. Sever and P. Vieira, Tailoring Three-Point Functions and Integrability III. Classical Tunneling, JHEP 07 (2012) 044 [arXiv:1111.2349] [INSPIRE].

[8] O. Foda, N=4 SYM structure constants as determinants, JHEP 03 (2012) 096 [arXiv: 1111.4663] [INSPIRE].

[9] I. Kostov, Classical Limit of the Three-Point Function of $N=4$ Supersymmetric Yang-Mills Theory from Integrability, Phys. Rev. Lett. 108 (2012) 261604 [arXiv:1203.6180] [INSPIRE].

[10] I. Kostov, Three-point function of semiclassical states at weak coupling, arXiv:1205.4412 [INSPIRE].

[11] V. Kazakov, A. Marshakov, J. Minahan and K. Zarembo, Classical/quantum integrability in AdS/CFT, JHEP 05 (2004) 024 [hep-th/0402207] [INSPIRE].

[12] B. Sutherland, Low-Lying Eigenstates of the One-Dimensional Heisenberg Ferromagnet for any Magnetization and Momentum, Phys. Rev. Lett. 74 (1995) 816 [INSPIRE]. 
[13] O. Foda and M. Wheeler, Partial domain wall partition functions, JHEP 07 (2012) 186 [arXiv: 1205.4400] [INSPIRE].

[14] L. Faddeev and L. Takhtajan, Spectrum and scattering of excitations in the one-dimensional isotropic Heisenberg model, J. Sov. Math. 24 (1984) 241 [INSPIRE].

[15] N.A. Slavnov, The algebraic Bethe ansatz and quantum integrable systems, Russian Mathematical Surveys 62 (2007) 727.

[16] R.J. Baxter, Exactly Solved Models in Statistical Mechanics, Academic Press, New York, U.S.A. (1982).

[17] V. Korepin and P. Zinn-Justin, Inhomogeneous Six-Vertex Model with Domain Wall Boundary Conditions and Bethe Ansatz, nlin/0008030.

[18] A.G. Izergin, Partition function of the six-vertex model in a finite volume, Soviet Physics Doklady 32 (1987) 878.

[19] A.G. Izergin, D.A. Coker and V.E. Korepin, Determinant formula for the six-vertex model, J. Phys. A 25 (1992) 4315.

[20] M. Gaudin, Boze Gas in One Dimension. II. Orthogonality of the Scattering States, J. Math. Phys. 12 (1971) 1677.

[21] K. Sogo, Time-Dependent Orthogonal Polynomials and Theory of Soliton - Applications to Matrix Model, Vertex Model and Level Statistics, J. Phys. Soc. Japan 62 (1993) 1887.

[22] O. Foda, M. Wheeler and M. Zuparic, XXZ scalar products and KP, Nucl. Phys. B 820 (2009) 649 [arXiv:0903.2611].

[23] O. Foda and G. Schrader, XXZ scalar products, Miwa variables and discrete KP, arXiv: 1003.2524.

[24] P. Zinn-Justin, Six-Vertex, Loop and Tiling models: Integrability and Combinatorics, arXiv:0901.0665.

[25] F. Colomo, A.G. Pronko and P. Zinn-Justin, LETTER: The arctic curve of the domain wall six-vertex model in its antiferroelectric regime, J. Stat. Mech.-Theory E. 3 (2010) L2 [arXiv: 1001.2189].

[26] O. Foda and M. Wheeler, Slavnov determinants, Yang-Mills structure constants and discrete $K P$, arXiv: 1203.5621 [INSPIRE].

[27] K. Takasaki, KP and Toda tau functions in Bethe ansatz, arXiv:1003.3071 [INSPIRE].

[28] M. Jimbo, T. Miwa and F. Smirnov, Hidden Grassmann Structure in the XXZ Model III: Introducing Matsubara direction, J. Phys. A 42 (2009) 304018 [arXiv:0811.0439] [InSPIRE].

[29] V.V. Bazhanov, T. Lukowski, C. Meneghelli and M. Staudacher, A Shortcut to the Q-Operator, J. Stat. Mech. 1011 (2010) P11002 [arXiv:1005.3261] [INSPIRE].

[30] N. Gromov and P. Vieira, Quantum Integrability for Three-Point Functions, arXiv:1202.4103 [INSPIRE].

[31] D. Serban, A note on the eigenvectors of long-range spin chains and their scalar products, arXiv:1203.5842 [INSPIRE].

[32] L. Faddeev, How algebraic Bethe ansatz works for integrable model, in proceedings of Les Houches Summer School, Session LXIV, NATO ASI Series Elsevier, Amsterdam (1998), pg. 149-220, hep-th/9605187 [INSPIRE].

[33] A. Faribault and D. Schuricht. On the determinant representations of Gaudin models' scalar products and form factors, arXiv:1207.2352. 